\title{
Modification of the Microstructure and Magnetic Properties of Ga-Ion-Irradiated Co/Pd Multilayer Film for Future Planar Media
}

\author{
E. Suharyadi ${ }^{a}$, S. Natsume ${ }^{a}$, T. Kato ${ }^{a}$, S. Tsunashima ${ }^{a}$, and S. Iwata ${ }^{b}$ \\ ${ }^{a}$ Department of Electronics, Graduate school of Engineering, Nagoya University, \\ ${ }^{\mathbf{b}}$ Center for Cooperative Research in Advanced Science and Technology (CCRAST), Nagoya University \\ Furo-cho, Chikusa-ku, Nagoya 464-8603, Japan
}

$\mathrm{Ga}$ ion irradiation was used to modify the microstructures and magnetic properties of $\mathrm{Co} / \mathrm{Pd}$ multilayers, and also to fabricate patterned media. A decrease in the diffraction peak at low-angle XRD showed that the regular interface between $\mathrm{Co}$ and $\mathrm{Pd}$ layers was blurred by ion irradiation. The effective perpendicular magnetic anisotropy energy, $K_{\text {eff }}$, decreased from $1.7 \times 10^{6}$ $\mathrm{erg} / \mathrm{cm}^{3}$ for as-grown film to $0.4 \times 10^{6} \mathrm{erg} / \mathrm{cm}^{3}$ for irradiated film at $2 \times 10^{15} \mathrm{ions} / \mathrm{cm}^{2}$. The easy axis of magnetization changed from perpendicular to in-plane at the highest ion dose $\left(5 \times 10^{15}\right.$ ions $\left./ \mathrm{cm}^{2}\right)$. An MFM image showed that the domain width gradually decreased with increasing ion dose, which corresponds to a decrease in the perpendicular magnetic anisotropy. Sharp boundaries between perpendicular and in-plane regions are also observed in patterned films, which indicates that localized magnetically patterns can be engineered by $\mathrm{Ga}$ ion irradiation.

Key words: planar media, $\mathrm{Co} / \mathrm{Pd}$ multilayer film, ion irradiation, perpendicular magnetic anisotropy, magnetic domain

\section{Introduction}

In the last several years, the rapidly growing interest in nano-scale magnetic thin film and patterned recording media based on lithographically patterned structures has made them promising base elements for future magnetic recording media ${ }^{1-5)}$, since the increasing density of conventional recording media will be limited by superparamagnetic behavior. Arrays for patterning the magnetic structure may be fabricated by lithography techniques such as etching, lift-off, and electroplating ${ }^{6-8)}$. However, to define the patterning arrays of the data bits, these techniques change the surface roughness of the magnetic media, and they have other drawbacks such as poor planarity. This would be a problem in hard disk recording technology, where the head flies at very high speed only $10 \mathrm{~nm}$ above the surface of the recording medium.

It has recently been reported that magnetic patterning of continuous magnetic film can be achieved by ion beam irradiation without significant modification of the surface roughness of the magnetic films ${ }^{9)}$. Chappert et al. first reported planar-patterned $\mathrm{Co} / \mathrm{Pt}$ magnetic multilayer films, where the He ion irradiation technique was used to induce local chemical mixing in a $\mathrm{Co} / \mathrm{Pt}$ multilayer film ${ }^{9)}$. The patterned magnetic structure resulted from the modulated distribution of chemical mixing, without any significant change in the topography. Using a different dose of high-energy ions, the researchers were also able to effectively reduce the coercivity of $\mathrm{Co} / \mathrm{Pt}$ multilayers. At a sufficiently high $\mathrm{He}$ ion dose of $10^{16}$ ions $/ \mathrm{cm}^{2}$, the easy axis of $\mathrm{Co} / \mathrm{Pt}$ multilayers is rotated from perpendicular into the plane of the film.

$\mathrm{Co} / \mathrm{Pd}$ multilayer films have also been extensively studied, since they exhibit large perpendicular magnetic anisotropy (PMA) and high coercivity ${ }^{10-12)}$. It has been reported that ion irradiation caused a decrease in the uniaxial magnetic anisotropy of $\mathrm{Co} / \mathrm{Pd}$ multilayer films ${ }^{13)}$. However, no systematic studies have yet been reported of the process for fabricating planar-patterned magnetic film in $\mathrm{Co} / \mathrm{Pd}$ multilayer film or of the possible correlation between the magnetic properties of irradiated $\mathrm{Co} / \mathrm{Pd}$ multilayer films and their microstructure, nor has there been any investigation of how isolated pattern magnetic domain structures can appear in planar-patterned media. Since the irradiated area has the same magnetization as the non-irradiated area, the exchange coupling between the bits may become important when ion irradiation is used to separate bits on the media. In this paper, the effects of $\mathrm{Ga}$ ion irradiation on the magnetic properties of $\mathrm{Co} / \mathrm{Pd}$ multilayer are investigated. $\mathrm{Ga}$ ion irradiation produced by a focused ion beam (FIB) is an excellent tool for fabricating a patterned magnetic structure without modification of the sample topography. The influence of ion irradiation on the magnetic properties and microstructure of $\mathrm{Co} / \mathrm{Pd}$ multilayer films and the modification of the magnetic properties of $\mathrm{Co} / \mathrm{Pd}$ multilayer films by $\mathrm{Ga}$ ion irradiation correlated with domain structure are discussed and analyzed in detail. An array of squares of $\mathrm{Co} / \mathrm{Pd}$ multilayer film with perpendicular magnetic anisotropy was also patterned by FIB, to study the feasibility of using such arrays in planar patterned media.

\section{Experimental method}

$\mathrm{Pd}(10 \mathrm{~nm}) /[\mathrm{Co}(0.4 \mathrm{~nm}) / \operatorname{Pd}(1.35 \mathrm{~nm})]_{20} / \mathrm{SiN}(70 \mathrm{~nm})$ films were prepared on a silicon substrate by RF magnetron sputtering. The background pressure in the sputtering system was below $3.0 \times 10^{-7}$ Torr. The $\mathrm{Ar}$ 
pressure during the sputtering of the $\mathrm{Co} / \mathrm{Pd}$ multilayer films was set at $20 \mathrm{mTorr}$. The $\mathrm{Co} / \mathrm{Pd}$ multilayer films were irradiated with a $22 \mathrm{keV} \mathrm{Ga}{ }^{+}$focused ion beam (FIB) at various ion irradiation doses from $5 \times 10^{12}$ to $5 \times 10^{15}$ $\mathrm{Ga}$ ions $/ \mathrm{cm}^{2}$ in order to study the corresponding changes in the magnetic properties of irradiated magnetic films. The ion irradiation was performed at room temperature with the $\mathrm{Ga}$ ion beam normal to the film surface. The $M-H$ loops of the sheet films were measured with an alternating gradient magnetometer (AGM). The magnetic anisotropies of films were measured with a torque magnetometer. Irradiated $\mathrm{Co} / \mathrm{Pd}$ multilayer films with sides $3 \mathrm{~mm}$ square and $10 \mathrm{~mm}$ square were prepared for AGM, torque magnetometer, and X-ray diffraction (XRD) measurement. The influence of the ion dose on the domain structure was studied by examining magnetic force microscopy (MFM) images. The surface profile was determined by atomic force microscopy (AFM). The $10 \times 10 \mu \mathrm{m}^{2}$ squares were irradiated for AFM and MFM measurement. The layered and crystalline structures were analyzed by the $\mathrm{X}$-ray diffraction (XRD) technique, using $\mathrm{Cu} K_{\alpha}(40 \mathrm{kV}, 30 \mathrm{~mA})$ radiation.

Patterned $\mathrm{Co} / \mathrm{Pd}$ samples were also fabricated by FIB etching at ion irradiation doses of $5 \times 10^{15}$ ions $/ \mathrm{cm}^{2}$. In the patterned films, un-irradiated squares with a size of $1 \mu \mathrm{m}$ were separated by irradiated regions with a size of $0.7 \mu \mathrm{m}$ to study the located magnetic domain structures of the planar-patterned magnetic media.

\section{Results and discussion}

\subsection{Microstructure and magnetic properties}

Fig. 1 shows the etching depth estimated from the AFM image as a function of the $\mathrm{Ga}$ ion dose. The etching depth of films irradiated with the maximum ion dose of 1 $\times 10^{16}$ ions $/ \mathrm{cm}^{2}$ was about $5.4 \mathrm{~nm}$. Since the thickness of the Pd capping layer is $10 \mathrm{~nm}$, only the Pd capping layer is assumed to be etched by the FIB irradiation, and the $\mathrm{Co} / \mathrm{Pd}$ magnetic multilayer film is not thought to be oxidized or etched.

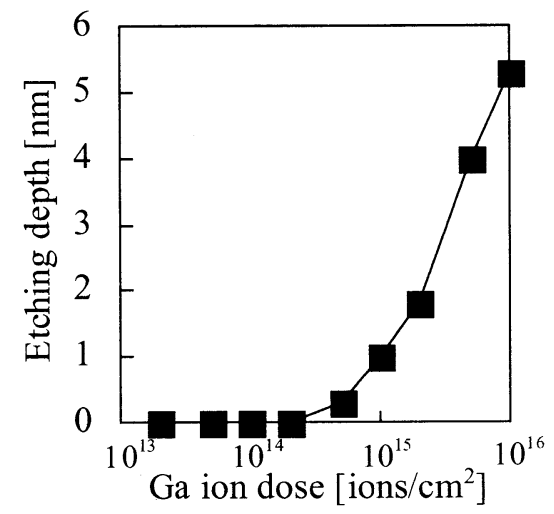

Fig. 1. Variation of the etching depth estimated by AFM result as a function of the $\mathrm{Ga}^{+}$ions dose. The zero ion dose is for as-grown film.

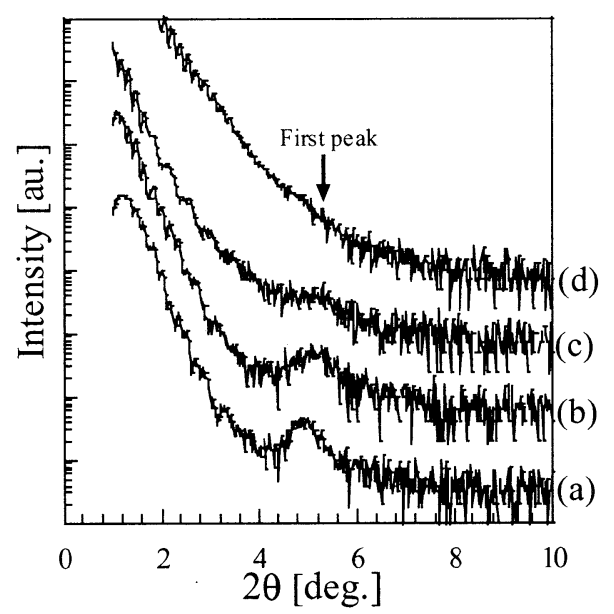

Fig. 2. Low-angle X-ray diffraction profiles of as-grown film (a) and irradiated films with ion doses of $2 \times 10^{13}(\mathrm{~b})$, $1 \times 10^{14}(\mathrm{c})$, and $5 \times 10^{14}$ ions $/ \mathrm{cm}^{2}(\mathrm{~d})$.

Fig. 2 shows a low-angle X-ray diffraction profile of as-grown and irradiated $\mathrm{Co} / \mathrm{Pd}$ multilayer films with various $\mathrm{Ga}$ ion doses. The low-angle X-ray diffraction profile is useful for determining the regularity of the periodic structure of $\mathrm{Co} / \mathrm{Pd}$ multilayers. The first peak indicated in Fig. 2 is attributed to the periodicity of the bilayer in the $\mathrm{Co} / \mathrm{Pd}$ multilayer film, which corresponds to the first-order diffraction of $\mathrm{Co} / \mathrm{Pd}$ bilayers with a periodicity of $1.76 \mathrm{~nm}$ as compared to the nominal periodicity of $1.75 \mathrm{~nm}$. The different first peak intensities of the four samples mainly reflect the difference in the interlayer structure of the $\mathrm{Co} / \mathrm{Pd}$ multilayer film. As-grown film exhibited a well-defined periodic structure and regular interface, as shown in the greater first peak intensity. It is known that the regular interface in the multilayers promotes a surface magnetic anisotropy in the easy axis perpendicular to the film plane. The first peak was decreased and broadened by increasing the ion irradiation dose, and disappeared at $5 \times 10^{14} \mathrm{ions} / \mathrm{cm}^{2}$. These results suggest that high-energy $\mathrm{Ga}$ ion irradiation diminishes the regular interfaces between Co and Pd layers in $\mathrm{Co} / \mathrm{Pd}$ multilayer films, on account of intermixing of atomics in the layers. The energy transferred to the multilayers via ion irradiation may affect the local atomic displacements and the interdiffusion of $\mathrm{Co}$ and $\mathrm{Pd}$ atoms.

Fig. 3 shows the $M-H$ loops of as-grown and irradiated $\mathrm{Co} / \mathrm{Pd}$ multilayer films when a magnetic field is applied perpendicular and parallel to the film plane. As-grown $\mathrm{Co} / \mathrm{Pd}$ multilayer film was confirmed to exhibit a square-shaped $M-H$ loop and display perpendicular easy magnetization with a remanence ratio of about 1 and a coercivity value of $1.45 \mathrm{kOe}$, as shown in Fig. 3(a). The as-grown sample showed an $H \mathrm{c}$ value of $1.45 \mathrm{kOe}$, but it has a rather large uniaxial anisotropy of $2 \times 10^{6} \mathrm{erg} / \mathrm{cc}$. Differences in the square-shaped $M-H$ loops were found among irradiated films, and the perpendicular remanence was decreased by increasing the ion irradiation dose, as shown in Fig. 3(b)-(d). It is assumed that a change in the 
microstructure leads to the modification of the hysterisis loop after irradiation. The coercive force and the remanence ratio are very sensitive to aspects of the local structure of the multilayer film, such as defects, the grain size, and the periodic stacking structure. For ion doses below $5 \times 10^{14}$ ions $/ \mathrm{cm}^{2}$, irradiated films had almost perfectly square perpendicular hysteresis loops, but the coercive force gradually decreased with increasing ion dose, and then abruptly decreased for films irradiated with an ion dose of $5 \times 10^{14} \mathrm{ions} / \mathrm{cm}^{2}$ at $H_{\mathrm{c}}=0.67 \mathrm{kOe}$ and $M_{\mathrm{r}} / M_{\mathrm{s}}=0.83$, as shown in Fig. 4 , and the magnetic anisotropy changed from the perpendicular to the in-plane direction at the highest ion dose, as shown in Fig. 3(d). The modification of the easy axis of the magnetic anisotropy following the ion irradiation is similar to the result previously reported by Chappert for ion-irradiated $\mathrm{Co} / \mathrm{Pt}$ multilayer films ${ }^{9)}$, and is thought to be responsible for the reduction of the interfacial anisotropy.

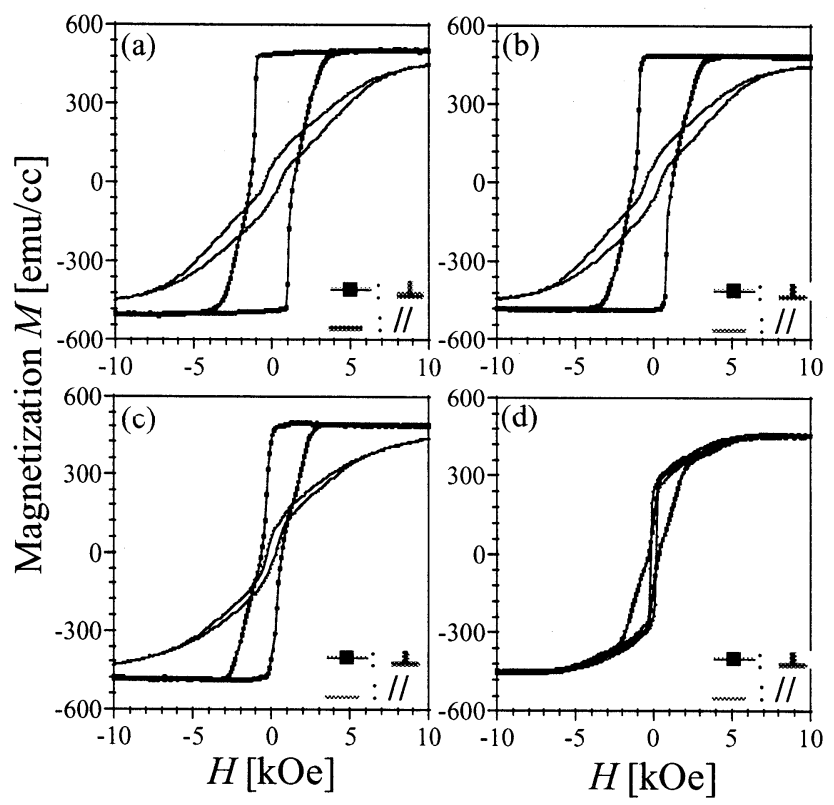

Fig. 3. Comparison of $M-H$ loops for as-grown film (a), and irradiated films with various ion doses, $2 \times 10^{13}$ (b), 5 $\times 10^{14}(\mathrm{c})$, and $5 \times 10^{15}$ ions $/ \mathrm{cm}^{2}(\mathrm{~d})$.

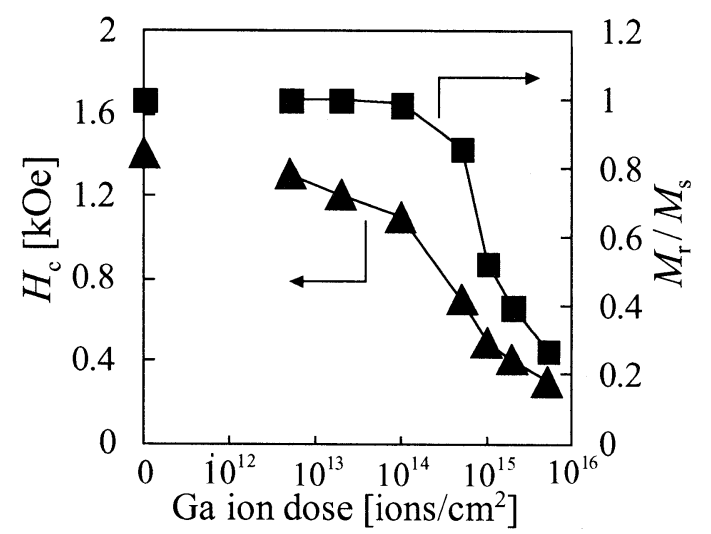

Fig. 4. Variation of the coercive field $(\Delta)$ and remanence ratio ( $\boldsymbol{\square})$ as a function of the $\mathrm{Ga}^{+}$ion dose. The zero ion dose is for as-grown film.

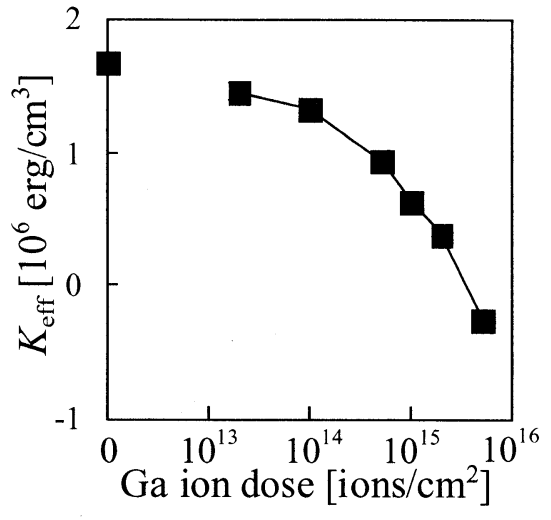

Fig. 5. Variation of the effective magnetic anisotropy energy $\left(K_{\text {eff }}\right)$ as a function of the $\mathrm{Ga}^{+}$ion dose. The zero ion dos is for as-grown film.

The magnetic anisotropy of the $\mathrm{Co} / \mathrm{Pd}$ multilayered film has been reported to be expressible as the sum of the interface anisotropy between Co and Pd layers and the crystalline and shape anisotropies of the Co layer. Carcia et al. introduced the following phenomenological equation involving the interface magnetic anisotropy of the $\mathrm{Co} / \mathrm{Pd}$ layer and the volume anisotropy of the Co layer, $K_{\text {eff }}=K_{\mathrm{v}}$ $+2 K_{\mathrm{s}} / t_{\mathrm{Co}}$, where $K_{\mathrm{eff}}$ is the effective uniaxial magnetic anisotropy for the unit volume of the Co layer, $K_{\mathrm{v}}$ is the volume anisotropy of the Co layer, $K_{\mathrm{s}}$ is the interface magnetic anisotropy between the Co and Pd layers, and $t_{\mathrm{Co}}$ is the thickness of the Co layer ${ }^{10)}$. Here, the value of $K_{\mathrm{v}}$ includes the contribution of the demagnetizing energy, and the positive values of $K_{\text {eff }}, K_{\mathrm{v}}$, and $K_{\mathrm{s}}$ represent the perpendicular magnetic anisotropy. Fig. 5 shows the change in the effective magnetic anisotropy energy $\left(K_{\text {eff }}\right)$ estimated from the amplitude of the torque curve for as-grown and irradiated films. In this investigation, the value of the effective magnetic anisotropy energy $\left(K_{\text {eff }}\right)$ is given for a unit volume of the $\mathrm{Co} / \mathrm{Pd}$ multilayer film. The interface anisotropy contribution, $K_{\mathrm{s}}$, seems to dominate in as-grown film and is then reduced in irradiated films because of the interdiffusion of $\mathrm{Co}$ and $\mathrm{Pd}$ atoms in $\mathrm{Co} / \mathrm{Pd}$ multilayer films. The change in the interface magnetic anisotropy caused by $\mathrm{Ga}$ ion irradiation is considered to be the main reason for the decrease in the effective magnetic anisotropy energy, $K_{\text {eff }}$, of $\mathrm{Co} / \mathrm{Pd}$ multilayer films, because the magnetization, $M_{\mathrm{s}}$, of as-grown films was almost constant after irradiation, as shown in Fig. 3. The effective perpendicular magnetic anisotropy energy, $K_{\text {eff, was }}$ gradually decreased by increasing the $\mathrm{Ga}$ ion irradiation from $1.7 \times 10^{6} \mathrm{erg} / \mathrm{cm}^{3}$ for as-grown film to $0.4 \times 10^{6}$ $\mathrm{erg} / \mathrm{cm}^{3}$ for film irradiated at $2 \times 10^{15} \mathrm{ions} / \mathrm{cm}^{2}$. The easy axis of magnetization changed from the perpendicular to the in-plane direction in film irradiated with an ion dose of around $5 \times 10^{15}$ ions $/ \mathrm{cm}^{2}$, since the effective magnetic anisotropy energy became negative, as shown in Fig. 5. It is assumed that the ion-beam-induced atomic disordering of the interface during ion irradiation, as clarified in low-angle X-ray diffraction profiles, is responsible for the changing magnetic anisotropy of $\mathrm{Co} / \mathrm{Pd}$ multilayer films. 
However, the low-angle first peak of films irradiated with an ion dose of $5 \times 10^{14}$ ions $/ \mathrm{cm}^{2}$ disappeared, but the effective magnetic anisotropy energy, $K_{\text {eff, was still }}$ positive at $0.9 \times 10^{6} \mathrm{erg} / \mathrm{cm}^{3}$, indicating a perpendicular easy axis. Another possibility is that the interdiffusion of $\mathrm{Co}$ and $\mathrm{Pd}$ atoms in $\mathrm{Co} / \mathrm{Pd}$ multilayer film after irradiation may change the $\mathrm{Co} / \mathrm{Pd}$ structure into a $\mathrm{CoPd} / \mathrm{Pd}$ structure, because the thickness of the Pd layer is much larger than that of the Co layer. Consequently, a low-angle first peak will be weakened in such a structure. But it should be noted that the effective magnetic anisotropy energy of $\mathrm{CoPd} / \mathrm{Pd}$ multilayer structures was still positive ${ }^{14)}$. It is considered that multilayer structures still exist in that film. At an ion dose of $5 \times 10^{14} \mathrm{ions} / \mathrm{cm}^{2}$, ion irradiation not only enhanced the interdiffusion between the $\mathrm{Co}$ and $\mathrm{Pd}$ layers but also increased the roughness of the interface, resulting in a decrease of the low-angle diffraction peak.
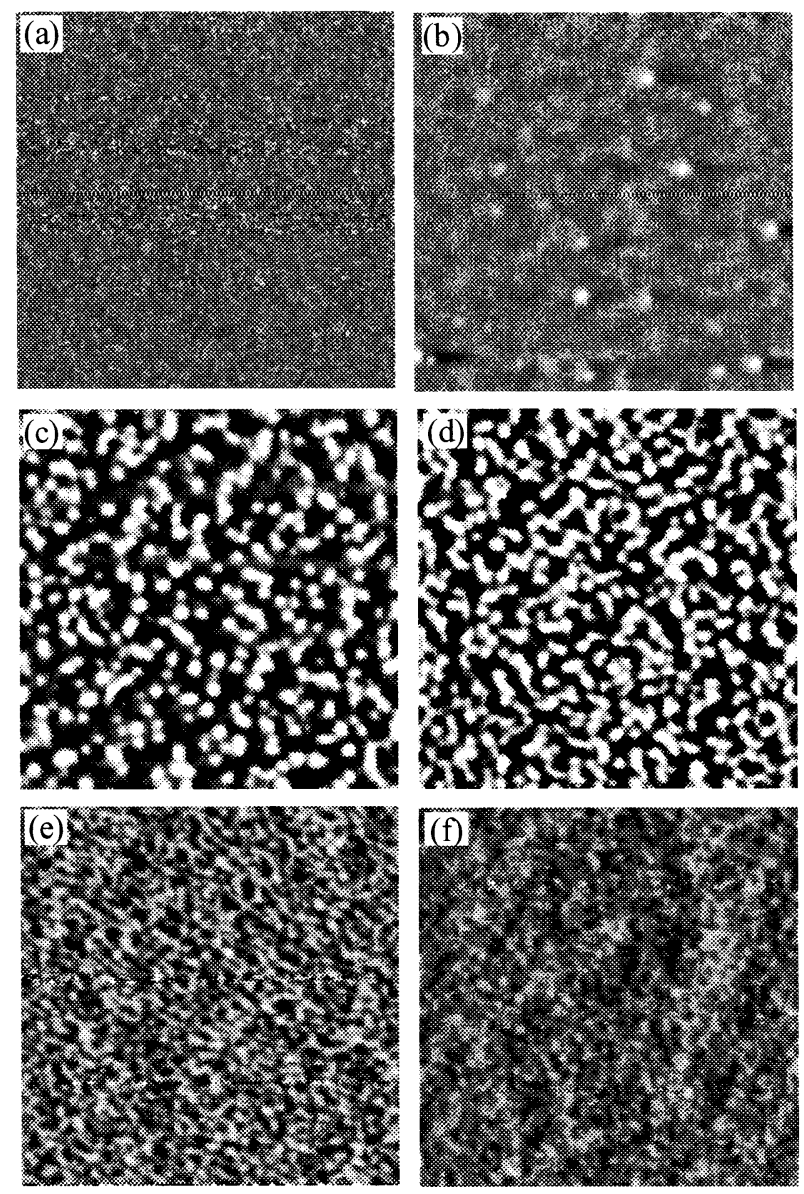

$1 \mu \mathrm{m}$

Fig. 6. MFM image in the remanent state after application of an external magnetic field of $15 \mathrm{kOe}$ along the surface normal direction: as-grown film (a), and after ion irradiation with $1 \times 10^{14}$ ions $/ \mathrm{cm}^{2}$ (b), $5 \times 10^{14}$ ions $/ \mathrm{cm}^{2}$ (c), $1 \times 10^{15}$ ions $/ \mathrm{cm}^{2}$ (d), $2 \times 10^{15}$ ions $/ \mathrm{cm}^{2}$ (e), and $5 \times$ $10^{15}$ ions $/ \mathrm{cm}^{2}$ (f).
It has been stated that ion irradiation reduces perpendicular anisotropy, and it is expected that ion irradiation will also change the magnetic domain structure. The effects of ion irradiation on the magnetic domain structure were studied for as-grown and irradiated films. Fig. 6 shows typical MFM images of domain structures of as-grown and irradiated $\mathrm{Co} / \mathrm{Pd}$ films in the remanent state. Here, the lift scan height during measurement was $50 \mathrm{~nm}$. We magnetized as-grown and irradiated $\mathrm{Co} / \mathrm{Pd}$ multilayer films once by applying a strong external magnetic field (15 kOe) in the direction normal to the film plane, and obtained MFM images in a zero field. The MFM tip was magnetized in the $z$ direction (perpendicular to the plane of the sample) prior to the measurement, and the magnetic contrast can be explained by an interaction between the perpendicular magnetized $\mathrm{Co} / \mathrm{Pd}$ multilayers and the MFM tip. As-grown film had a uniform contrast, as shown in Fig. 6(a). This result suggests that the remanent state of as-grown film can be described as a single magnetic domain, because it has large remanent magnetization almost equal to the saturation magnetization, as shown in Fig. 3(a). In film irradiated with an ion dose of $1 \times 10^{14}$ ions $/ \mathrm{cm}^{2}$ (Fig. 6(b)), some bright spots were clearly seen, which may reflect a transition from a single-domain into a maze-domain structure. The maze-domain structure was clearly seen in irradiated films, as shown in Fig. 6(c)-(f). In irradiated films, the typical maze-domain structure still had a similar irregular appearance when the ion irradiation dose was increased, but the magnetic domain size decreased. It has been noted that width and separation of the domain structures is determined by the ratio of the magnetic wall energy $\sqrt{2 A_{0} K_{\text {eff }}}$ to the demagnetization energy, $2 \pi M_{\mathrm{s}}^{2}$, where $A_{0}$ is the exchange constant, $K_{\text {eff }}$ is the effective magnetic anisotropy energy, and $M_{\mathrm{s}}$ is the saturation magnetization ${ }^{15)}$. A substantial decrease in magnetic domain size was evident when the ion irradiation dose was increased. Since the magnetization $M_{\mathrm{s}}$ of as-grown and irradiated films was almost the same, a decrease in the magnetic domain size is due to a decrease in the domain wall energy, which corresponds to a decrease in the effective magnetic anisotropy, as confirmed in Fig. 5. The contrast decreased with increasing ion irradiation doses and with the resulting decrease in the domain size. In films irradiated with ion doses of $2 \times 10^{15} \mathrm{ions} / \mathrm{cm}^{2}$ and $5 \times 10^{15} \mathrm{ions} / \mathrm{cm}^{2}$, the contrast decreased especially significantly. This might be due to a decrease in the size of the perpendicularly magnetized domains.

From the observed stripe domain width of $\sim 200 \mathrm{~nm}$ for the sample dosed at $1 \times 10^{15}$ ions $/ \mathrm{cm}^{2}$, as shown in Fig. 6(d), the domain wall energy was estimated to be $\sim 6$ $\mathrm{erg} / \mathrm{cm}^{2}$ for this sample, assuming the demagnetizing energy of parallel band domains in a thin perpendicular magnetized film ${ }^{16)}$. The uniaxial anisotropy $K_{\mathrm{u}}$ (which is $K_{\text {eff }}+2 \pi M_{\mathrm{s}}^{2}$ ) was $2.1 \times 10^{6} \mathrm{erg} / \mathrm{cc}$. Thus, by assuming the simple expression $4 \sqrt{A_{0} K_{\mathrm{u}}}$ for the energy density of Bloch walls and neglecting the demagnetizing energy in the wall, the exchange stiffness $A_{0}$ is estimated to be $1.1 \times$ 
$10^{-6} \mathrm{erg} / \mathrm{cm}$. If we use these values and assume the simple expression $\pi \sqrt{A_{0} / K_{\mathrm{u}}}$ for the domain wall width, the wall width can be estimated at $\sim 20 \mathrm{~nm}$ for a sample irradiated with an ion dose of $1 \times 10^{15}$ ions $/ \mathrm{cm}^{2}$. The estimated domain walls are considered to give a measure of the space (transitional space) between patterned bits in planar patterned $\mathrm{Co} / \mathrm{Pd}$ media.

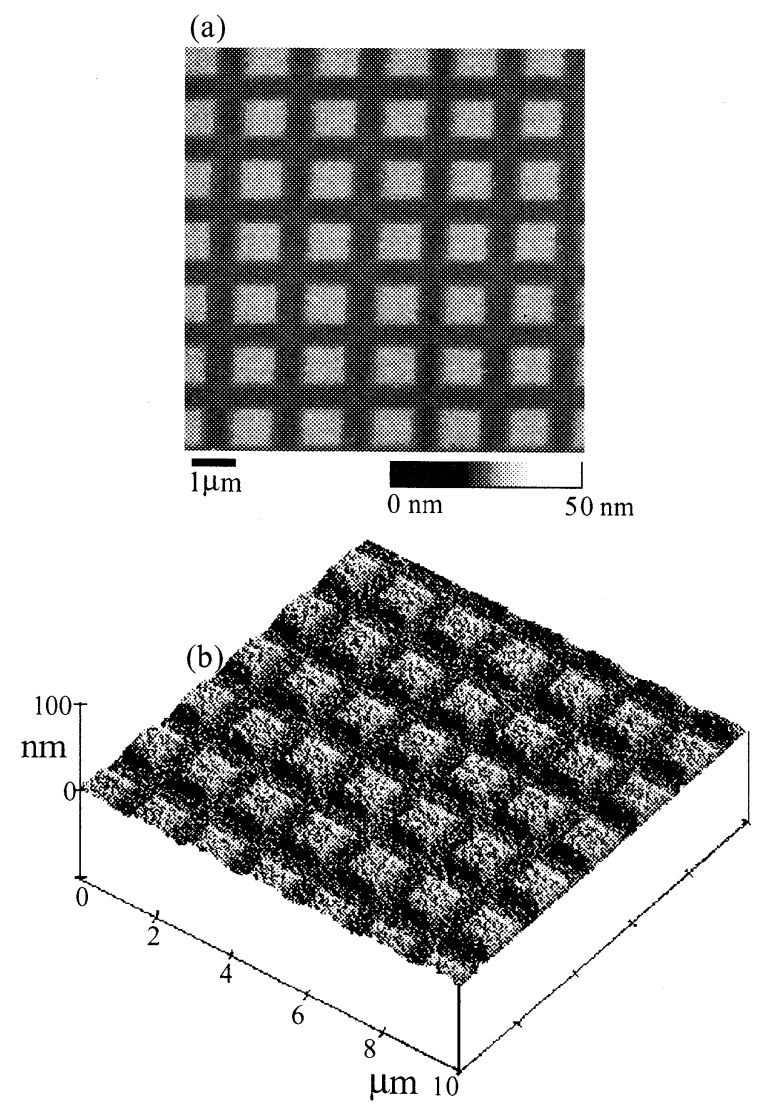

Fig. 7. AFM images of an array of squares $1 \mu \mathrm{m}$ in size with a spacing of $0.7 \mu \mathrm{m}$ : plane view (a) and 3-dimensional view (b).

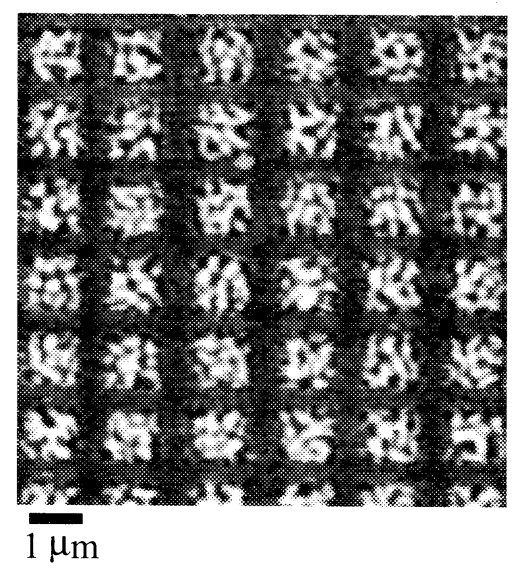

Fig. 8. MFM image of ion-irradiation-patterned films with a pattern size of $1 \mu \mathrm{m}$ and a spacing of $0.7 \mu \mathrm{m}$.

\subsection{Ion-irradiation-patterned Co/Pd multilayers}

Fig. 7 shows AFM images of ion-irradiation-patterned $\mathrm{Co} / \mathrm{Pd}$ multilayer films irradiated at an ion dose of $5 \times 10^{15}$ ions $/ \mathrm{cm}^{2}$. The etching depth of the irradiated region is about $4.5 \mathrm{~nm}$. The ion-irradiation-patterned $\mathrm{Co} / \mathrm{Pd}$ film was smooth, showing regions of $10 \mu \mathrm{m} \times 10 \mu \mathrm{m}$, where squares with a size of $1 \mu \mathrm{m} \times 1 \mu \mathrm{m}$ were separated by a distance of $0.7 \mu \mathrm{m}$, as shown in Fig. 7(a). In addition, the surface roughness of ion-irradiation-patterned film is an important property. Three-dimensional AFM images of patterned media give further insight into modification at the surfaces, as shown in Fig. 7(b). An MFM image of a patterned film is shown in Fig 8 . This image was taken without any external magnetic field applied after the sample deposition. The significant difference in magnetic contrast and typical domain structures between un-irradiated and irradiated regions can be clearly observed in the patterned film. The MFM image of the patterned film indicates that each un-irradiated square consists of localized maze domain structures, which corresponds to perpendicular magnetization direction. Low magnetic contrast and the absence of maze structures in irradiated areas may be due to the change of the magnetization direction from perpendicular to in-plane. The boundary between the perpendicular region and in-plane region is very sharp. It is considered that the $\mathrm{Ga}$ ion beam induces atomic disordering and separates the magnetized regions, which may be useful for separating bits on media.

\section{Conclusion}

We have demonstrated that $\mathrm{Ga}$ ion irradiation modified the magnetic properties and microstructures of $\mathrm{Co} / \mathrm{Pd}$ multilayer films. The effect of an ion dose on the layered structure was confirmed by low-angle XRD measurement. The first peak due to the multilayer structure disappeared after irradiation with an ion dose of $5 \times 10^{14}$ ions $/ \mathrm{cm}^{2}$. Ion irradiation had a significant influence on the regularity of the periodic structure in $\mathrm{Co} / \mathrm{Pd}$ multilayer films because of the interdiffusion between $\mathrm{Co}$ and $\mathrm{Pd}$ atoms at the interface. The modifications of magnetic properties by $\mathrm{Ga}$ ion irradiations were associated with a decrease in the interfacial magnetic anisotropy. At lower $\mathrm{Ga}$ ion doses $(<$ $5 \times 10^{14}$ ions $/ \mathrm{cm}^{2}$ ), the multilayer film retained its perpendicular anisotropy, but the coercive force gradually decreased. The transition from perpendicular to in-plane direction occurred at a $\mathrm{Ga}$ ion irradiation dose of around 2 $\times 10^{15}$ ions $/ \mathrm{cm}^{2}$. A further decrease in the perpendicular magnetic anisotropy was observed at higher Ga ion doses. MFM images of the irradiated films showed that the domain width gradually decreased with increasing ion dose. Significant differences in magnetic contrast and domain structure between irradiated and un-irradiated regions were observed in the patterned films. A sharp boundary between perpendicular and in-plane regions was also observed, indicating that localized patterns can be engineered by $\mathrm{Ga}$ ion irradiation. 
Acknowledgements The authors would like to thank Mr. M. Kumazawa of Nagoya University for experimental assistance.

\section{References}

1) C. A. Ross, H. I. Smith, T. Savas, M. Schattenburg, M. Farhoud, M. Hwang, M. Walsh, M. C. Abraham, and R.J . Ram: J. Vac. Sci. Technol. B 173168 (1999).

2) C. A. Ross: Annu. Rev. Mater. Res. 31203 (2001).

3) R. M. H. New, R. F. W. Pease, and R.L. White: $J$. Magn. Magn. Mater. 155140 (1996).

4) M. Todorovic, S. Schultz, J. Wong, and A. Scherrer: Appl. Phys. Lett. 742516 (1999).

5) G. F. Hughes: IEEE Trans. Magn. 36521 (2000).

6) M. Hehn, K. Ounadjela, J. P. Bucher, F. Rousseaux, D. Decanini, B. Bartenlian, and C. Chappert: Science 272 1782 (1996).

7) P. B. Fischer, M. S. Wei, and S. Y. Chou: J. Vac. Sci. Technol. B 112570 (1993).

8) P. R. Krauss, P. B. Fischer, and S. Y. Chou: J. Vac. Sci. Technol. B 123639 (1994).
9) C. Chappert, H. Bernas, J. Ferre, V. Kottler, J.-P. Jamet, Y. Chen, E. Cambril, T. Devolder, F. Rousseaux, V. Mathet, and H. Launois: Science 2801919 (1998).

10) P. F. Carcia, A. D. Meinhaldt, and A. Suna: Appl. Phys. Lett. 47178 (1985).

11) S. Hashimoto and Y. Ochiai: J. Magn. Magn. Mater. 88211 (1990).

12) J. M. MacLaren and R. H. Victoria: App. Physics Lett. 663377 (1995).

13) F. J. A. den Broeder, D. Kuiper, and H. J. G.. Draaisma: IEEE Trans. Mag. 233696 (1987).

14) S. Tsunashima, K. Nakamura, T. Takahashi, S. Fukatsu, and S. Uchiyama: Mat. Res. Soc. Symp. Proc. 231441 (1992).

15) J. A. Cape and G. W. Lehman: J. Appl. Phys. 425732 (1971).

16) A. Hubert and R. Schäfer: Magnetic Domains: The Analysis of Magnetic Microstructure, Springer Verlag ,Berlin, Heidelberg, pp. 291-354 (1998).

Received Mar. 14, 2005; Accepted June 10, 2005. 